\title{
HIV-1 and methamphetamine alter galectins $-1,-3$, and -9 in human monocyte-derived macrophages
}

\author{
Kinga Grabowska ${ }^{1,2} \cdot$ Katarzyna Macur $^{1,3} \cdot$ Sarah Zieschang $^{1} \cdot$ Lubaba Zaman $^{1} \cdot$ Nicole Haverland ${ }^{1}$. \\ Andrew Schissel ${ }^{1} \cdot$ Brenda Morsey $^{1} \cdot$ Howard S. Fox $^{1} \cdot$ Pawel Ciborowski $^{1}$
}

Received: 5 July 2021 / Revised: 30 September 2021 / Accepted: 27 October 2021 / Published online: 17 February 2022

(c) The Author(s) 2022

\begin{abstract}
Macrophages are key elements of the innate immune system. Their HIV-1 infection is a complex process that involves multiple interacting factors and various steps and is further altered by exposure of infected cells to methamphetamine (Meth), a common drug of abuse in people living with HIV. This is reflected by dynamic changes in the intracellular and secreted proteomes of these cells. Quantification of these changes poses a challenge for experimental design and associated analytics. In this study, we measured the effect of Meth on expression of intracellular and secreted galectins-1, -3 , and -9 in HIV-1 infected human monocyte-derived macrophages (hMDM) using SWATH-MS, which was further followed by MRM targeted mass spectrometry validation. Cells were exposed to Meth either prior to or after infection. Our results are the first to perform comprehensive quantifications of galectins in primary hMDM cells during HIV-1 infection and Meth exposure a building foundation for future studies on the molecular mechanisms underlying cellular pathology of hMDM resulting from viral infection and a drug of abuse-Meth.
\end{abstract}

Keywords Galectins · HIV · Innate immunity · Macrophages · Multiple reaction monitoring · Quantitative proteomics

\section{Introduction}

HIV-1 infection has devastating effects at various levels on the function of the entire organism (Niu et al. 2020; Passaro et al. 2015; Saito et al. 2008). The macrophage (mononuclear phagocytes, MP), a key component of the innate immune system, is one of the prime targets of HIV-1 and a reservoir of productive viral infection constituting a vehicle to spread infection to organs. Thus, its impact on the course of disease

Kinga Grabowska and Katarzyna Macur contributed equally to this work.

Pawel Ciborowski

pciborowski@unmc.edu

1 Department of Pharmacology and Experimental Neuroscience, School of Medicine, University of Nebraska Medical Center, Omaha, NE, USA

2 Laboratory of Virus Molecular Biology, Intercollegiate Faculty of Biotechnology, University of Gdańsk and Medical University of Gdańsk, Gdańsk, Poland

3 Core Facility Laboratories, Intercollegiate Faculty of Biotechnology, University of Gdańsk and Medical University of Gdańsk, Gdańsk, Poland is central. The complexity of HIV infection and treatment is further exacerbated by using drugs of abuse because two entities (virus and drug of abuse) are involved, the virus and use of drugs of abuse. Treatments are quite different in nature (Liang et al. 2008). Meth also has untoward effects on various physiological systems including the immune system (Papageorgiou et al. 2019) and is one of the frequently used drugs in people living with HIV (PLWH) (Lyons et al. 2013; Mitchell et al. 2006; Pantalone et al. 2010).

MPs survey their milieus and react to contain their pathological environment, including infections, or other sources of inflammation. However, exposure to toxic substances, including Meth, impairs their protective capacities. It has been shown that HIV-1 infection results in changes in MP protein expression (Talloczy et al. 2008); and therefore, the addition of Meth may further impact the functioning of the innate immune system (Kraft-Terry et al. 2009).

Galectins are defined as lectins binding $\beta$-galactoside. There have been 15 galectins discovered in mammals, out of which nine, galectin- $1,-2,-3,-4,-7,-8,-9,-10$, and -12 , have been found in humans (https://www.genenames.org/data/ genegroup/\#!/group/629). Because carbohydrate-protein interactions are generally weak (Laederach and Reilly 2005), 
dissociation constants for most lectin-monosaccharide interactions are in the millimolar range (Schwarz et al. 1993). It has been shown that the galectin-1 dimer is the most thermodynamically stable of all galectins with dimer dissociation constant of $\mathrm{K}_{\mathrm{d}} \sim 2-7 \times 10^{-6} \mathrm{M}$. Based on biochemical characterization of lectins, galectins, as well as other lectins, enable the formation of transient states, which might support interactions with protein as their ligands (Schwarz et al. 1993).

Although galectins have been postulated as central regulators of the immune system (Brinchmann et al. 2018; Paclik et al. 2011), the mechanism of how they regulate monocyte/ macrophage physiology, which constitute the connection between innate and adaptive branches of the immune system, is still unknown. Paclik et al. postulate that galectins uniquely modulate central monocyte/macrophage function (Paclik et al. 2011). The authors showed that galectins link the innate and adaptive immune systems by inhibiting T-cell function via macrophage priming.
As depicted in Fig. 1a, galectins are divided to three subgroups: prototype, chimera type, and tandem repeat type. Galectin-1, galectin-3, and galectin-9 represent prototype, chimeric, and tandem repeat groups, respectively. Regardless of their structure, galectins participate in regulation of many cellular functions in macrophages as presented in Fig. 1b. These are, but not limited to, adhesion and migration, apoptosis, cell transformation, tumor growth, angiogenesis, and immune escape. A wide variety of biological processes have been reported to be related to galectins, such as development, differentiation, morphogenesis, tumor metastasis, apoptosis, and RNA splicing. Nevertheless, the mechanism by which galectins use carbohydrate recognition for their functions is not fully understood.

As galectins themselves and their involvement in HIV-1 infection have been studied to some extent (Shahbaz et al. 2020), the effect of Meth on their expression is represented by very few publications (Parikh et al. 2015; Reynolds et al. 2007). In this study, we present results of the
Fig. 1 Galectins and their functions in macrophages. (a) Types of galectins. (b) Summary of functions of galectins in macrophage (adapted from (Johannes et al. 2018; Vladoiu et al. 2014))
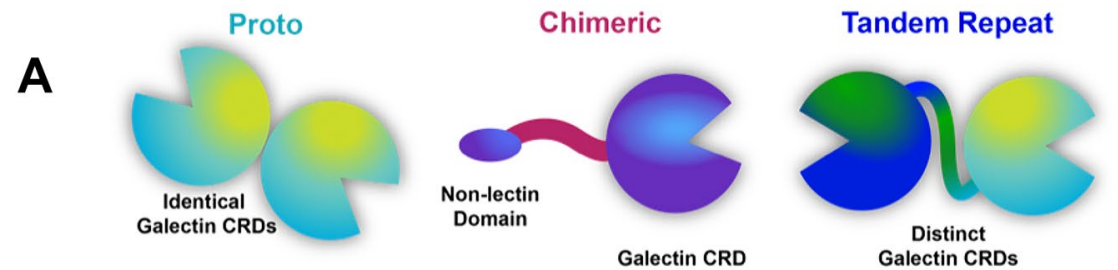

B
Extracellular galectins

PMID: 12223274
Intracellular galectins

PMID: 19594636

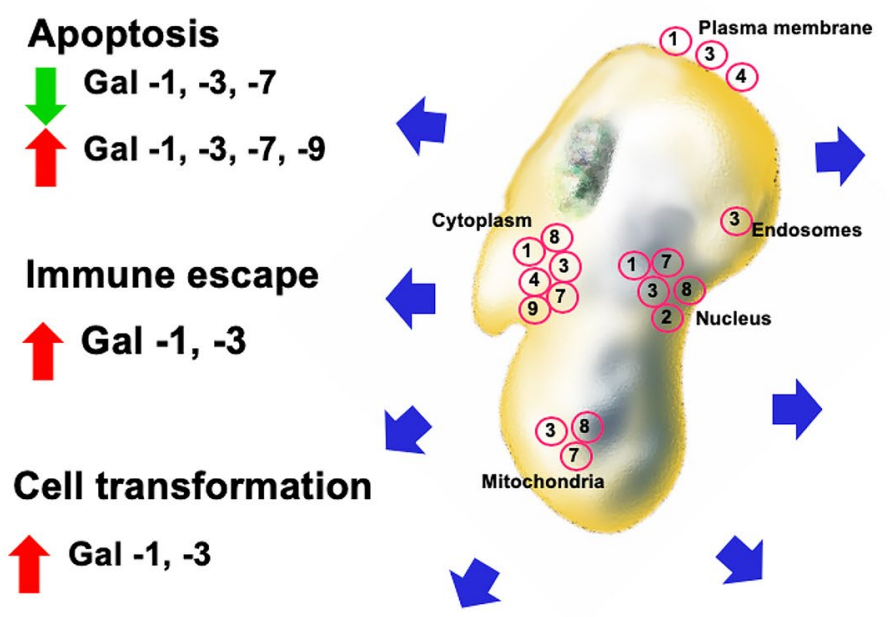

\section{Tumor growth Gal -7 \\ Gal $-1,-3,-4,-7$, $-8,-9,-12$}

\section{Angiogenesis}

Gal -7

Gal -1, -3
Invasion \& metastasis

Gal -7

Gal -3, $-4,-9$ 
effect of Meth on intracellular and extracellular expression of galectins-1, -3, and -9 in HIV-1 infected human monocytederived macrophages (hMDM). Each galectin represents different sub-type of this molecule. Interestingly, we demonstrate that high sensitivity and accuracy methods such as mass spectrometry-based multiple reaction monitoring (MRM) show significant differences in expression, which are not readily detected by screening methods such as sequential window acquisition of all theoretical mass spectra (SWATH-MS).

Galectins are soluble proteins found primarily in the cytosol, nucleus, extracellular matrix, or in circulation. Our immunocytochemical staining shows that an intracellular pool of these three galectins is divided between lysosomes and cytosol. This suggests that each pool of galectin plays different role(s) in cells' metabolism.

\section{Materials and methods}

\section{Patients and samples}

As reported before (Haverland et al. 2014; Macur et al. 2021), monocytes from healthy human donors were obtained from HIV-1, HIV-2, and hepatitis seronegative donors and differentiated to hMDM. The University of Nebraska Medical Center (UNMC) Institutional Review Board has determined utilizing core facilities from leukapheresis of normal donors does not constitute human subject research as defined in 45CFR46.102 of US Federal Policy for the Protection of Human Subjects. Therefore, this leukapheresis procedure is not subject to federal regulation of human subject research and has been classified as exempt.

\section{Cell cultures and infections}

Samples were prepared as described in Macur et al. (2021) . Leukapharesed monocytes purified by counter-current centrifugal elutriation were resuspended in the serumfree Macrophage-SFM media (Life-Tech, Inc., Houston, TX, USA) supplemented with 10-mM HEPES (Invitrogen, Carlsbad, CA, USA), $50 \mathrm{mg} / \mathrm{mL}$ gentamicin (Invitrogen), $2 \mu \mathrm{g} / \mathrm{mL}$ ciporflaxin (Invitrogen), $1 \%$ Nutridoma-SP (Sigma, St. Louis, MO, USA), and $10 \mathrm{ng} / \mathrm{mL}$ of recombinant human macrophage colony stimulating factor (MCSF; PeproTech, Rocky Hill, NJ, USA) and then seeded on 6 well culture plates. Cells were cultured for 7 days with half- and complete-media exchange on 3rd and 5th day, respectively, as well as with or without Meth (Sigma) treatment (Meth final concentration $100 \mu \mathrm{M}$ ). On 7th day post-elutriation, media were discarded, and differentiated macrophages were exposed for $4 \mathrm{~h}$ to $1 \mathrm{ml}$ of serum-free media containing $\mathrm{HIV}-1_{\mathrm{ADA}}\left(\mathrm{TCID}_{50}=10^{\wedge} 4.36\right.$ per $\mathrm{ml}$, as measured on human monocyte-derived macrophages). After 4-h exposure, all media containing HIV-1 ${ }_{\mathrm{ADA}}$ was removed, cells were washed with serum-free media four times, covered with the fresh serum-free MacrophageSFM media supplemented with 10-mM HEPES, 50-mg/mL gentamicin, $2-\mu \mathrm{g} / \mathrm{mL}$ ciporflaxin, and $1 \%$ Nutridoma-SP (without addition of MSCF) with or without Meth treatment and cultured for a total of 5 days post HIV- $1_{\mathrm{ADA}}$. On 3 rd day post-infection, a full media exchange (with or without Meth treatment) was performed and the supernatant reserved for a p24 assay (Macur et al. 2021). On 5th day post-infection, conditioned media was collected, spun at $1500 \mathrm{rpm}$ to remove any floating cells and major debris and transferred to the 50-ml tubes. Cells were washed with ice-cold Dulbecco's phosphate-buffered saline (DPBS; Corning, Manassas, VA, USA); and DPBS with the Halt ${ }^{\mathrm{TM}}$ Protease and Phosphatase Inhibitor Cocktail, EDTA-free (Thermo Fisher Scientific, Waltham, MA, USA), was added to each well. Each well was scraped; the cellular suspension was transferred to 15-mL tubes, washed in DPBS with the protease inhibitors, and pelleted by centrifugation. Cell pellets and collected conditioned media of HIV-1 uninfected/Meth untreated (CCC), infected with HIV-1 (CIC), exposed to Meth postinfection (CIM), and treated with Meth before and after HIV-1 infection (MIM) were stored at $-80^{\circ} \mathrm{C}$ for further analysis. For further details see Fig. 2.

\section{RNA isolation and RNAseq}

For RNA isolation, the culture medium was removed; cells were washed with ice-cold DPBS; and attached hMDMs were dissolved in Trizol (Invitrogen) and stored at $-80^{\circ} \mathrm{C}$. Trizol MDM extracts were subsequently thawed; chloroform was added to each tube, mixed, incubated at room temperature for $10 \mathrm{~min}$, and further separated by centrifugation. The aqueous phase was transferred to a new tube, and RNA was precipitated by adding isopropyl alcohol then incubated for 20 min at $-20{ }^{\circ} \mathrm{C}$. RNA was then recovered by centrifugation at $12,000 \mathrm{~g}$ at $4{ }^{\circ} \mathrm{C}$ for $10 \mathrm{~min}$, and the pellet dissolved in RNAse-free water. The RNAeasy clean-up kit (Qiagen, Germantown, MD, USA) was used for cleaning the RNA preparation. Quantity and purity were quantified by absorbance measurement at $260 \mathrm{~nm}$ and 230/280 $\mathrm{nm}$ absorbance, respectively. Intactness of prepared samples was assessed on a Fragment Analyzer (Agilent Technologies, Ankeny, IA, USA).

The RNA samples then were used for cDNA library construction using TrueSeq mRNA sample prep V2 (Illumina, San Diego, CA, USA) followed by next-generation sequencing performed on the Illumina NextSeq550. The sequencing data was processed and mapped to the human reference genome as described (Macur et al. 2021). For expression calculation and ANOVA analysis, values of Transcripts Per Kilobase Million (TPM) were calculated; 


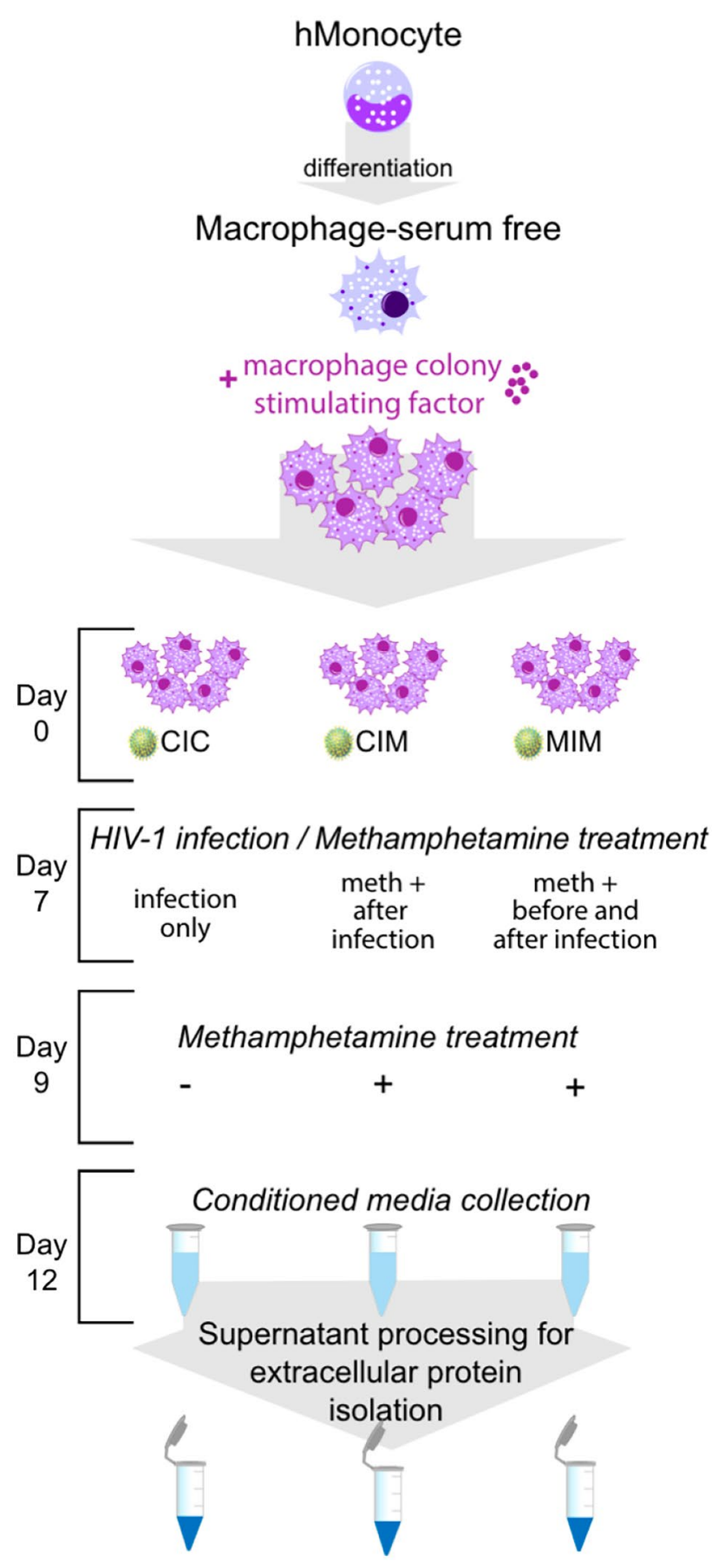

Fig. 2 Experimental design. CIC signifies condition in which infection only occurs; CIM represents Meth treatment post-infection. MIM signifies Meth treatment before and after infection. An addition of Meth prior to and after infection (MIM) and after infection (CIM) indicates longer and shorter time of exposure to Meth, respectively

and data were imported into the Partek Flow genomic analysis software package (Partek Inc., St. Louis, MO, USA), followed by batch correction for donors using a generalized linear model and statistical analysis by ANOVA.

\section{Cell lysates and extracellular proteins from conditioned media}

Cell lysis was performed as described in our other papers (Burns and Ciborowski 2016; Macur et al. 2021). Samples were digested using the Filter Aided Sample Preparation (FASP) protocol (Wisniewski et al. 2009). Obtained peptides were cleaned by applying Oasis mixed cation exchange procedure according to the manufacturer's protocol (Waters, Milford, MA, USA). Peptides concentration was measured using a NanoDrop 2000 (Thermo Scientific, Wilmington, DE, USA). Collected supernatants were thawed, supplemented with protease/phosphatase inhibitor cocktail, Triton X-100 (Fisher Scientific, Pittsburg, PA, USA) at a final concentration of $0.1 \%$ and after incubation centrifuged at $1500 \mathrm{rpm}$ for $10 \mathrm{~min}$. New supernatants were transferred to the $50 \mathrm{~mL}$ tubes and concentrated using Amicon®Ultra-15 centrifugal filter units (Millipore, Bedford, MA, USA) with a membrane cutoff of 3000 MWCO (molecular weight cutoff). Concentrated supernatants were processed for ethanol precipitation procedure, followed by albumin//gG depletion using Pierce ${ }^{\mathrm{TM}}$ Albumin/IgG Removal Kit (Thermo Fisher Scientific). For total protein concentrations measurement Pierce $660 \mathrm{~nm}$ Protein Assay (Thermo Fisher Scientific) was applied.

\section{Liquid chromatography-tandem mass spectrometry analysis}

\section{NanoLC-SWATH-MS analyses}

A nanoLC-MS/MS (nano liquid chromatography-tandem mass spectrometry) technique in a data-independent acquisition (DIA) SWATH (Sequential Windowed Acquisition of All Theoretical Fragment Ion Mass Spectra) mode was applied for full unbiased proteomic profiling of CIC, CIM, and MIM samples from four donors, as described in Macur et al. (2021). The nanoLC-MS/MS system consisted of Eksigent 415 nanoLC (Eksigent, Redwood, CA, USA) coupled with TripleTOF 6600 (SCIEX, Framingham, MA, USA) mass spectrometer with ESI OptiFlow TurboV Ion Source and SteadySpray NanoProbe (SCIEX). The system was controlled by Analyst TF 1.7 software (SCIEX). The CIC, CIM, and MIM samples consisted of aliquots of trypsin-digested hMDM whole cell extracts from all the studied donors for each of the conditions. They were spiked with iRT Kit peptides (Biognosys AG, Zurich, Switzerland) and analyzed by SWATH-MS in five technical replicates. Peptide mixtures $(2 \mu \mathrm{g} /$ injection $)$ were loaded onto the RP-1 Trap Column (General RP, $10 \times 0.075 \mathrm{~mm}$; Phenomenex, Torrance, CA, USA) for $10 \mathrm{~min}(2 \mu \mathrm{L} / \mathrm{min}$ flow rate) and transferred to the bioZen Peptide Polar-C18 separation column $(150 \times 0.075 \mathrm{~mm}, 3 \mu \mathrm{m}$; Phenomenex $)$ 
through SecurityLINK Sgl $(25 \mu \mathrm{m} \times 75 \mathrm{~cm}$, Phenomenex $)$. The chromatographic separation was carried out using the mobile phases A $(0.1 \%$ formic acid in water) and B $(0.1 \%$ formic acid in acetonitrile (ACN) (all solvents LC-MS grade) at the flow rate of $300 \mathrm{~nL} / \mathrm{min}$. The gradient program started from 3 to $8 \%$ B (0-2 min), then linearly increased to $30 \% \mathrm{~B}(2-90 \mathrm{~min}), 40 \% \mathrm{~B}(90-100 \mathrm{~min})$ and $80 \%$ B (100-105 min); then it was maintained at $80 \%$ B (105-110 $\mathrm{min})$ and followed by column re-equilibration at 3\% B (112-135 min). The chromatographically separated samples were then ionized by ESI at $3 \mathrm{kV}$ ion spray voltage and analyzed by SWATH-MS in a positive ion mode for 130 min, where the TOF-MS MS1 scan in the $\mathrm{m} / \mathrm{z}$ range of $400-1000 \mathrm{Da}$ was divided into 100 overlapping variable precursor isolation windows and followed by MS 2 scans acquired in a looped product ion mode in $100-1500 \mathrm{~m} / \mathrm{z}$ range for +2 to +5 -charged precursors. A pooled CIC-CIM-MIM sample spiked with iRT Kit peptides was also analyzed in four technical replicates using data-dependent acquisition (DDA) in positive ion mode on the same nanoLC-MS/MS system, where up to 30 of the most intense precursor ions were selected from TOF-MS MS1 scan (400-1250 Da) for fragmentation in the $100-1500 \mathrm{~m} / \mathrm{z}$ range. The nanoLC and ion source setup in and parameters in DDA analyses were identical to the SWATH-MS analyses. The mass spectrometry DDA and SWATH data from the presented study were deposited to the ProteomeXchange Consortium (Perez-Riverol et al, 2019) (http://proteomecentral.proteomexchange.org) and are available under the PXD023291 dataset identifier. The obtained DDA MS/MS spectra of the pooled CIC-CIMMIM sample were searched together using ProteinPilot 5.0.1 software (SCIEX) with Paragon Algorithm against a human and HIV reviewed database from UniProt (https:// www.uniprot.org/) (downloaded on 09.04.2020) to create a spectral ion library. This spectral ion library (only peptides with confidence threshold of $99 \%$ and 1\% FDR rate, shared peptides excluded) was then applied for the SWATH data extraction with the use of SWATH 2.0 MicroApp in PeakView 2.2 software (SCIEX). The extracted peak areas for the proteins were then exported to MarkerView 1.2.1 software (SCIEX), normalized, and statistically analyzed using t-Test to compare CIC vs. CIM, CIC vs MIM, and CIM vs MIM conditions. The details of the nanoLC-MS/ MS DIA SWATH and DDA MS/MS methods' settings as well as parameters of the database search, SWTAH data extraction, and statistical analysis with the resulting $p$ values (statistical significance at $p<0.05$ ) and $\log _{10}$ (Fold Change) of proteins quantified in hMDM in CIC vs. CIM, CIM vs. MIM, and CIM vs. MIM conditions, can be found in Macur et al. (2021). Sequence coverage resulting from nanoLC MS/MS analysis for galectins-1, -3 , and -9 are shown in Supplemental Fig. S1.

\section{LC-MRM analyses}

A reverse phase ultra-high-performance LC-MS/MS technique in MRM mode was applied for quantification of galectins $(1,3,9)$ in five human donors' samples. The analyses were performed on the Nexera X2 UHPLC with a SIL30AC Autosampler (Shimadzu, Kyoto, Japan) coupled with the QTRAP 6500 mass spectrometer (SCIEX), which were controlled by Analyst software (SCIEX). A Phenomenex reverse phase HPLC Omega $(1.6 \mu \mathrm{m}$, PS C18, $100 \times 2.1 \mathrm{~mm}$, $100 \AA$ ) column with SecurityGuard ${ }^{\mathrm{TM}}$ ULTRA Cartridge was used for separation of the peptide mixtures resulting from trypsin digestion of CIC, CIM, and MIM whole cell extracts of hMDM, for analysis of intracellular galectins (2, 4 , and $6 \mu \mathrm{g}$ injections), or hMDM culture supernatants, for analysis of extracellular galectins ( $4 \mu \mathrm{g}$ injection). A linear gradient program from 5 to $50 \%$ solvent B for 25 min was applied for the peptides mixtures separation at the flow rate of $0.2 \mathrm{~mL} / \mathrm{min}$. Then the gradient was increased to $95 \% \mathrm{~B}$ for $3 \mathrm{~min}$ and followed by column re-equilibration for $10 \mathrm{~min}$, also at the flow rate of $0.2 \mathrm{~mL} / \mathrm{min}$. The solvent $A$ was water containing $0.1 \%$ formic acid, while solvent $\mathrm{B}$ was ACN containing $0.1 \%$ formic acid (all LC-MS grade). The eluting peptides were ionized by ESI in TurboV Ion Source (SCIEX) at a positive $5 \mathrm{kV}$ spray voltage and analyzed using MRM. The MRM transitions for galectin-1, -3 , and -9 were in silico predicted using Skyline software (MacLean et al. 2010) and then empirically verified with the use of the pooled donors' CIC-CIM-MIM hMDM sample. The parameters for the MRM transitions of the investigated galectins peptides are presented in the Table S1 (Supplementary Materials). Peak areas of the monitored MRM transitions from the LC-MRM raw data were extracted also using Skyline. They were used as an input data for the Excel package (Microsoft, Inc.) for calculation of their corresponding peptides, which were further calculated to the peak areas of the analyzed proteins. A two-sample unequal variance $\mathrm{T}$-test was performed using the $\log 2$ transformed protein peak areas to compare the investigated galectins levels between the studied conditions (CIC, CIM, and MIM). The differences were considered statistically significant when $p<0.05$. The MRM dataset from our study is available in PASSEL repository (PeptideAtlas SRM Experiment Library) (Farrah et al. 2012) under the accession number PASS01591. Detailed description of the MRM assay development with Skyline settings for transition prediction and the method validation as well as the parameters of the LC-MRM method and statistical analysis are available in Macur et al. (2021).

\section{Immunofluorescence confocal microscopy analysis}

Human monocytes were differentiated to macrophages as described above; on day 7 were exposed to Meth with the 
final concentration at $100 \mu \mathrm{M}$. Following 4-h incubation, cells were stained with LysoTracker® Red DND-99 (Thermo Fisher Scientific) according to the manufacturer's protocol. Next, cells were fixed with cold $4 \%$ paraformaldehyde in phosphate-buffered saline PBS and permeabilized with $0.2 \%$ (w/v) Triton X-100 in phosphate-buffered saline (PBS) for 8 min. After washing with PBS, cells were stained with rabbit anti-galectin-1 (ab25138) (Abcam, Cambridge, United Kingdom) Abs (1:500), mouse anti-galectin-3 (B2C10) (Santa Cruz Biotechnology, Dallas, TX, USA) Abs (1:500) or rabbit anti-galectin-9 (ab69630) (Abcam) Abs (1:200) for $1.5 \mathrm{~h}$. Next, cells were incubated with the suitable secondary antibodies Alexa 488-conjugated anti-mouse IgG (1:3000) or Alexa 488-conjugated anti-rabbit IgG (1:3000) (both Invitrogen) for $1 \mathrm{~h}$. For cell nuclei visualization, ProLong ${ }^{\mathrm{TM}}$ Gold antifade reagent with DAPI (Invitrogen) was applied. Prepared slides were analyzed using a ZEISS LSM 800 confocal laser scanning microscope (Zeiss, Jena, Germany).

\section{Results}

\section{Experimental design}

Our main question in this study was the effect of Meth on already HIV-1-infected macrophages, and if such effect exists, how much macrophages are further altered in their intra- and extracellular metabolism. Figure 2 reflects such objective; thus, our control was set to be HIV-1-infected macrophages. In addition to full unbiased profiling, we focused on galectins, both intracellular and secreted. Another question asked was whether exposure to Meth prior to HIV-1 infection has a different effect on hMDM than exposed to Meth only after viral infection (CIM) or continuously (MIM).

\section{Analysis of gene expression}

RNA was isolated from the cultures of six donors' MDM, cultured under the three conditions, and subjected to RNAseq. Examination of galectin-1, -3, and -9 expression levels did not reveal changes due to either Meth condition (Fig. 3).

\section{SWATH-MS analysis}

As depicted in Fig. 2, our experimental design set HIV1-infected hMDM (with no Meth treatment) as the control (CIC), experimental samples when Meth was added after viral infection as CIM, or both before and after infection as MIM. We applied nanoLC-SWATH-MS approach to obtain full unbiased proteomic profiles of hMDM in each of the studied conditions. This enabled us to see changes in the proteins' expression on the global level that occur

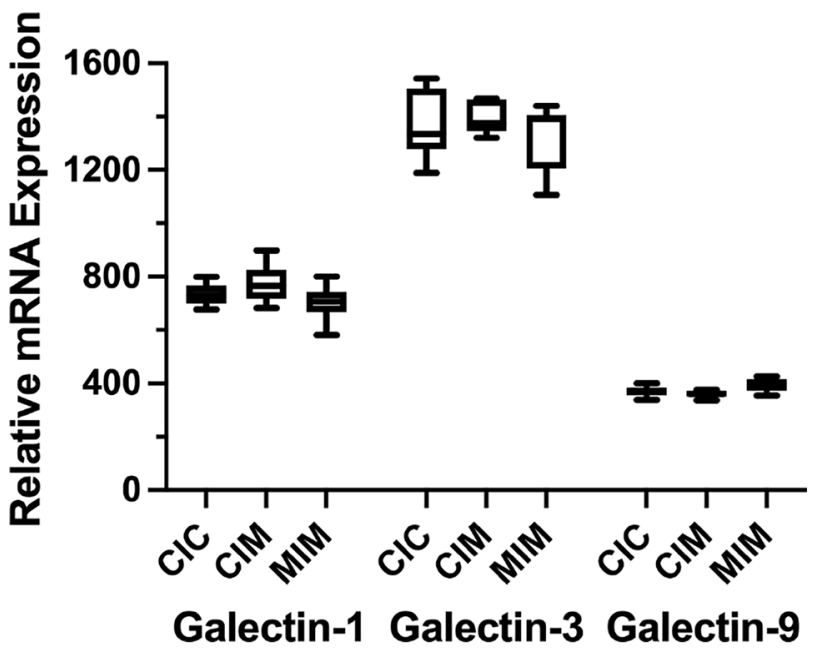

Fig. 3 Box and whisker plots of RNA-seq determination of expression levels of galectin-1, -3 , and -9 . No change was found between the conditions

as a consequence of HIV infection with simultaneous Meth treatment pre- and/or-post infection. In the SWATH experiment, we used monocytes from five donors (five biological replicates) that were analyzed in five technical replicates (five injections of each sample). Details of this SWATH-MS experiment were reported previously (Macur et al. 2021). Briefly, a reference spectral library for SWATH data extraction was generated experimentally using DDA mass spectrometry. We used non-fractionated trypsin digest of pooled CIC, CIM, and MIM samples from four donors to generate a library that contained 1241 proteins (1\% FDR). This library was then applied for SWATH data extraction. The obtained peak areas of proteins detected and quantified in our study for each of the investigated conditions (CIC, CIM, MIM) were then compared using $t$ test and $\log _{10}$ (Fold Change) between CIC versus CIM, CIC versus MIM, and CIM versus MIM were calculated. Table 1 shows this relative quantification and consists of data extracted from SWATH-MS experiment specifically for galectins $-1,-3$, and -9 .

\section{MRM analysis}

To verify our findings from the SWATH experiment, we quantified galectins- $1,-3$, and -9 using LC-MRM, a wellestablished MS-based approach for reproducible and sensitive quantification. MRM-based quantification is a more precise and accurate method than full unbiased SWATH-MS. In some instances, SWATH-MS does not detect some proteins while MRM can provide reproducible quantification. In this study, we used an MRM approach to validate and a more in-depth mass spectrometry-based analysis to validate the expression of three 
Table 1 Differences in expression of intracellular galectins identified and quantified by SWATH experiment in hMDM in CIC, CIM, and MIM conditions

\begin{tabular}{|c|c|c|c|c|c|c|c|}
\hline \multirow[t]{2}{*}{ UniProt accession number } & \multirow[t]{2}{*}{ Name } & \multicolumn{2}{|c|}{ CIC vs CIM } & \multicolumn{2}{|c|}{ CIC vs MIM } & \multicolumn{2}{|c|}{ CIM vs MIM } \\
\hline & & $p$ value & $\log _{10}($ Fold Change $)$ & $p$ value & $\log _{10}($ Fold Change $)$ & $p$ value & $\log _{10}($ Fold Change $)$ \\
\hline splP09382ILEG1_HUMAN & Galectin-1 & $6.40 \mathrm{E}-01$ & $2.78 \mathrm{E}-02$ & 4.39E-01 & $-5.87 \mathrm{E}-02$ & $2.32 \mathrm{E}-01$ & $-8.65 \mathrm{E}-02$ \\
\hline sp|P17931|LEG3_HUMAN & Galectin-3 & $9.12 \mathrm{E}-01$ & $-6.25 \mathrm{E}-03$ & $8.00 \mathrm{E}-01$ & $1.37 \mathrm{E}-02$ & 4.43E-01 & 1.99E-02 \\
\hline splO00182|LEG9_HUMAN & Galectin-9 & $6.96 \mathrm{E}-01$ & $-4.95 \mathrm{E}-02$ & 3.68E-01 & $-1.77 \mathrm{E}-01$ & $4.52 \mathrm{E}-01$ & $-1.27 \mathrm{E}-01$ \\
\hline
\end{tabular}

galectins representing three sub-types. At this stage, we measured both intra- and extracellular galectins levels in hMDM whole cell protein extracts and cell supernatants, respectively. We analyzed CIC, CIM, and MIM samples separately for 5 donors (each in 5 technical replicates). We developed the MRM assays for the investigated galectins with the aid of in silico MRM transitions prediction using Skyline software (MacLean et al. 2010). The theoretical transitions were then verified experimentally on a pooled CIC-CIM-MIM sample, to ensure reliable analysis of the target samples. The LC-MRM methodology was also validated using serial dilutions of bovine serum albumin standard spiked into the CIC-CIM-MIM sample matrix. The MRM transitions monitored for the galectin-1, -3 , and -9 are presented in Table S1 (Supplementary Material). The detailed information about LC-MRM assay development, validation, the LC-MS/MS settings, and data analysis is available in (Macur et al. 2021).

\section{Intracellular galectins}

Figure 4 shows MRM quantification-based effect of Meth on the intracellular galectins-1, -3 , and -9 . Our baseline is hMDM infected with HIV-1 without Meth (CIC). In this figure, we show results of quantification of galectins in hMDM from five donors. Four (D164, D172, D406, and D421) out of five donors show similar pattern of expression of Gal-1 in three experimental conditions. Only one donor (D222) shows a different pattern contributing to the variability of responses between donors, an effect observed by us in many experiments. Interestingly, Gal-3 does not show a consistent pattern among the same set of donors. Three (D164, D172, and D421) show similar patterns of Gal-9 expression, while two other donors (D406 and D222) show different patterns than the other three donors; however the pattern is similar for these two. Summarizing, the characteristics of protein expression in primary macrophages varies in different donors. We postulate those differences in patterns would be dependent on other factors, such as the donor and response to HIV infection. It has to be noted that samples for this study represent one time point, while the response of macrophages can be very dynamic in terms of quantity of changes. We also postulate that short-lived regulatory proteins might show highly diverse patterns.

\section{Extracellular galectins}

Concentrations of extracellular galectins are summarized in Fig. 5.

\section{Intra-cellular localization of galectins}

As shown in Fig. 6, localization of galectins in cells under the control condition is across entire cytoplasm with a concentration in the perinuclear region. There is no significant difference between patterns of intracellular distribution of the three galectins being investigated here.

To test cellular distribution of galectins- $1,-3$, and -9 as well as colocalization with acidic organelles-lysosomescytostaining of control and Meth exposed hMDM was performed. In both conditions, galectins colocalize with acidic organelles and are observed in the central part of the cell. Interestingly, this effect is more pronounced for galectin-9 of hMDM Meth-treated (Fig. 7).

\section{Ingenuity pathway analysis (IPA)}

Data obtained from MarkerView were used for comparisons of three biological conditions CIC vs CIM, CIC vs MIM, and CIM vs MIM and were uploaded to IPA as three separate observations. The data comprised of $\mathrm{p}$-value and $\log _{10}($ Fold Change) for each observation. Then, core analysis was run with preset analysis settings in which we specified analyzing all pathways for humans only. The results for all three observations were then run through comparison analysis in IPA. Insignificance threshold for $-\log (\mathrm{P}$-value $)$ was set to 1.5 for the outputs obtained. The three networks were finally overlapped to find connections between galectin-1, -3, and -9 (Fig. 9).

IPA analysis presented in Fig. 8 shows connection of galectins-1, -3 and -9 in their regulatory processes. The presented network shows that these galectins are connected as well as they are connected to a number of other regulatory proteins. One group consists of several chemokines such as CXCL17, CCL24, CCL25, and CCL28 associated primarily with galectin-1. These chemokines are involved in regulating, in positive or negative mode, T cells, macrophages, monocytes, and dendritic cells (DC), all relevant for the function of the immune system (Lee et al. 2013) as well as other cells of hematopoietic origin. 
Fig. 4 MRM quantification of intracellular expression of galectins $-1,-3$, and -9 under Meth treatment of HIV-1 infected hMDM. For quantification of each galectin, we used 2, 4, or 6 ug of cell lysates representing pool of intracellular proteins. Each condition was measured five times (five technical replicates). One star represents $\mathrm{p}<0.05$, two stars represent $\mathrm{p}<0.01$, and three stars represent $\mathrm{p}<0.001$

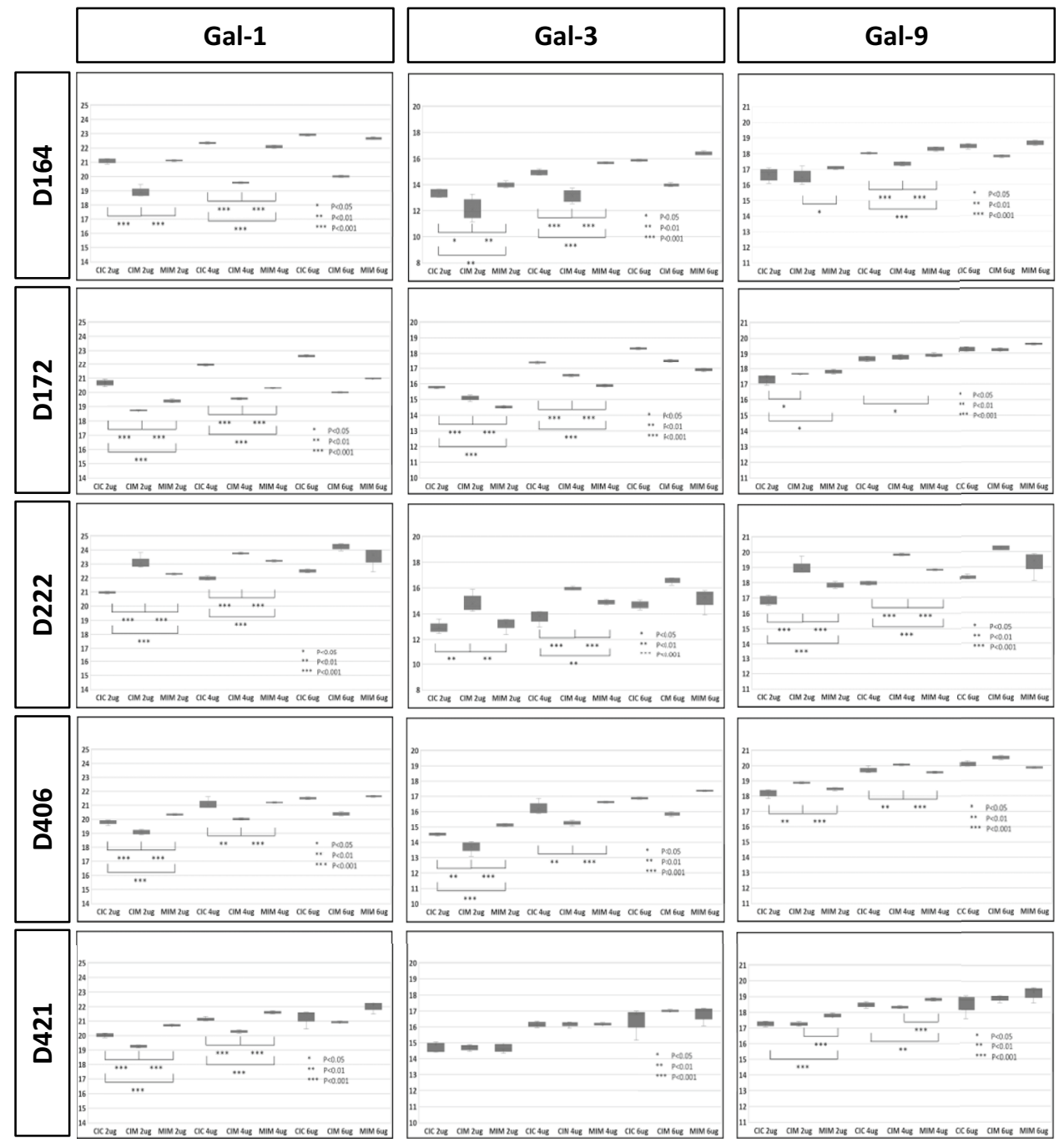

The same network shows several genes involved with ion transport (Lysosomal Solute Carrier Transporters) such as SLC9A6, SLC12A2, SLC12A7, SLC12A6, SLC12A4, and SLC38A9 that are associated primarily with galectin-9. SLC transporters have been shown as essential for ion, molecule, and other solute transport to support lysosomal function (Bissa et al. 2016). More specifically SLC38A9 mediates transport of amino acids with low capacity and specificity with a slight preference for polar amino acids. SLC12A2 cation-chloride cotransporter mediates the electroneutral transport of chloride, potassium, and/or sodium ions across the membrane. SLC9A6 electroneutral exchange of protons for $\mathrm{Na}^{+}$and $\mathrm{K}^{+}$across the early and recycling endosome membranes contributes to calcium homeostasis. SLC12A7 mediates electroneutral potassium-chloride cotransport when activated by cell swelling. SLC12A6 mediates electroneutral potassium-chloride cotransport. It is important to note that our cyto-immune staining shows some association of galectins with LysoTracker; however, Meth does not have a profound effect on such co-localization.
Another example is ALCAM (activated leukocyte cell adhesion molecule, Q13740, CD166_HUMAN) that is directly connected to all galectins being investigated here (Ikeda and Quertermous 2004). ALCAM is a cell adhesion molecule mediating cell-cell contacts. Galectin-3 is located in the middle/center of IPA-generated network and link Gal-1 and Gal-9.

Further IPA core analysis showed that canonical analysis indicated the most change as measured by fold change was the BEX2 signaling pathway. BEX2 is a regulator of mitochondrial apoptosis and G1 cell cycle in breast cancer (Naderi et al. 2010). This aligns with other reports showing the effect of Meth on mitochondrial function (Barbosa et al. 2015). Following canonical pathways, we present upstream regulators. The latter category shows the strongest effect of Meth on pro-inflammatory cytokines (Fig. 9b). Again, it aligns with previously reported observation showing pro-inflammatory response of macrophages exposed to Meth (Bortell et al. 2015; Burns and Ciborowski 2016). 
Fig. 5 MRM quantification of extracellular expression of galectins- $1,-3$, and -9 under Meth treatment of HIV-1-infected hMDM. For quantification of each galectin, we used 4 ug of cell lysates representing a pool of extracellular proteins. Each condition was measured five times (five technical replicates) in cultures of hMDM from five donors. One star represents $\mathrm{p}<0.05$, two stars represent $\mathrm{p}<0.01$, and three stars represent $\mathrm{p}<0.001$

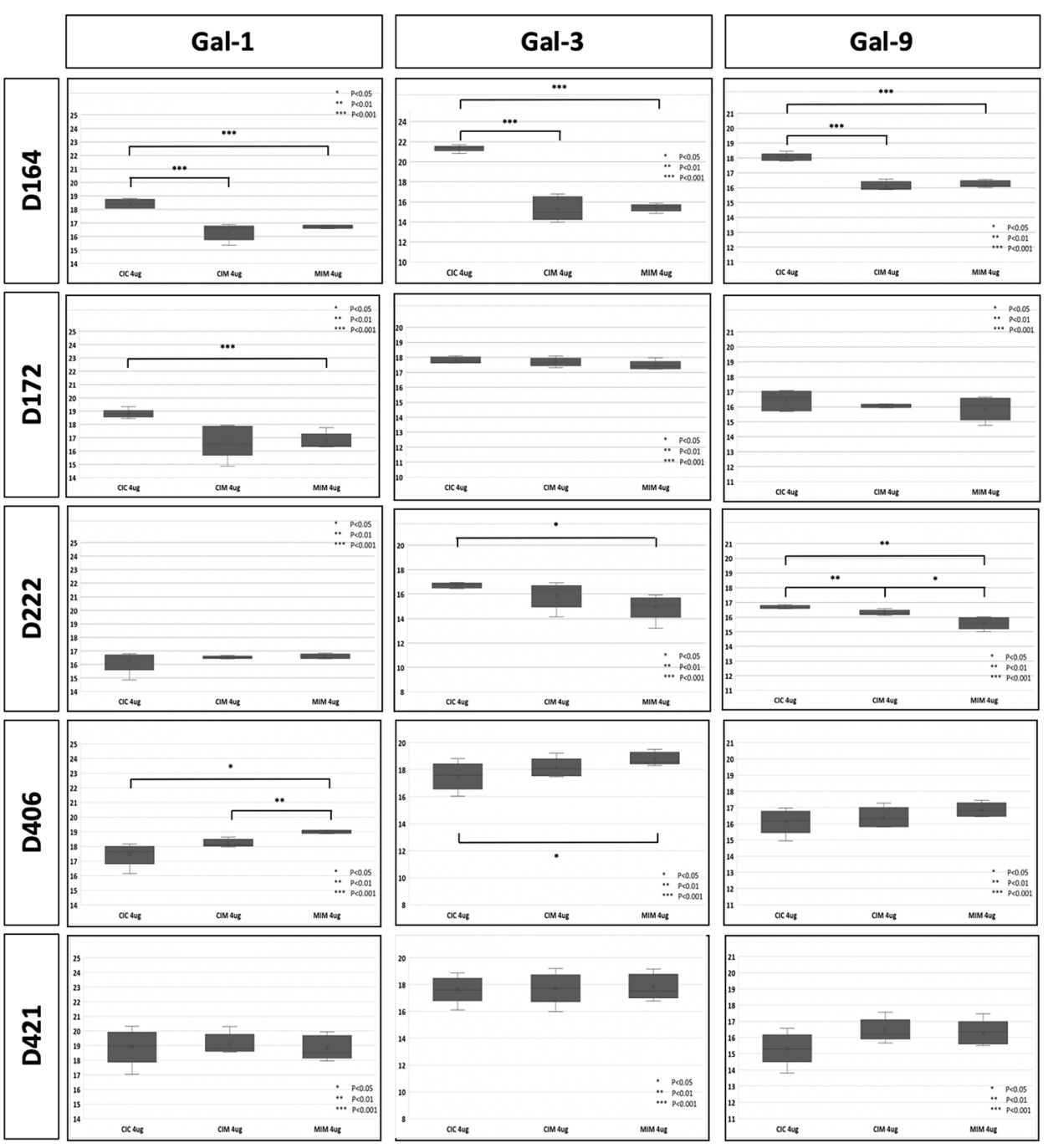

\section{Discussion}

As much as galectins themselves were studied to some extent, the effect of Meth on their expression is represented by very few publications (Parikh et al. 2015; Reynolds et al. 2012b, 2007). Published results do not allow for more comprehensive interpretation due to a variety of different experimental models used. In this study, based on
SWATH-MS, followed by MRM, we present results of the effect of Meth on intracellular expression of galectin-1, -3, and -9 in HIV-1-infected hMDM. Analytical measurements applied here appear to be precise enough to detect small but significant differences. Our previous study (Haverland et al. 2014) showed that Meth had profound and statistically significant effects on expression of nucleic acid binding and regulatory proteins in HIV-1 infected macrophages.
Fig. 6 Intracellular localization of galectins- $1,-3$, and -9 in hMDM. Immuno-histostaining for CD163 (green), galectins (red), and nuclei DAPI (blue). Galectins are distributed across entire cells; nevertheless, most of them are around nuclei
Galectin-1

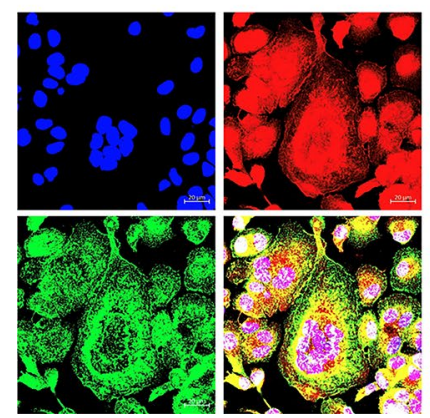

Galectin-3

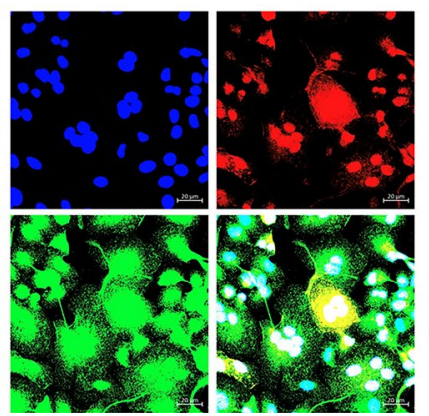

Galectin-9

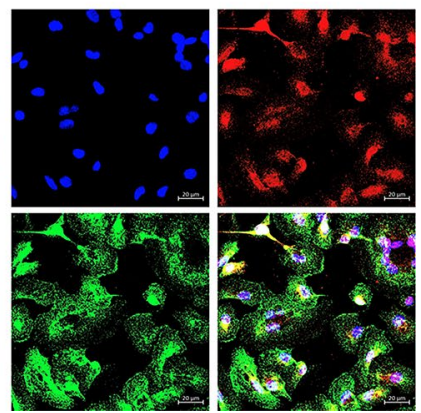


Fig. 7 Cytostaining of control and Meth exposed hMDM for galectins $-1,-3$, and -9 (green), acidic organelles - lysosomes (fluorescently labeled by lysotracker-red), and nuclei (DAPI-blue). A, B, E, F, I, and $\mathrm{J}$ represent single channel for lysosomes; and C, D, G, H, K, and $\mathrm{L}$ are merged images
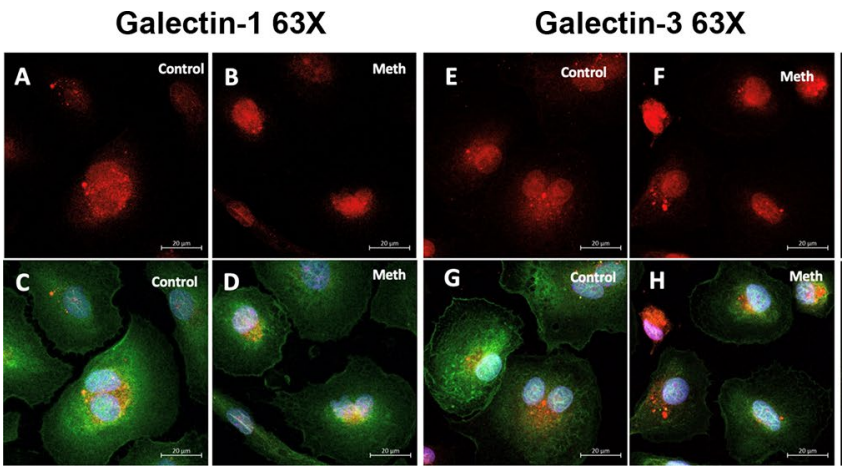

In that study, we used 4 donors of hMDM and performed SWATH-MS analysis using four groups: control, HIV-1 infected, Meth exposed, and HIV-1 infected and exposed to Meth; thus, experimental design was different than in this current one. Re-analyses of previous data are now included as Fig. S1 and Table S1 in the supplemental material.

Comparing previously published results (Haverland et al. 2014) and current SWATH-MS and MRM-MS analysis results, we conclude that SWATH-MS is substantially less sensitive and precise as MRM. Major limitation of MRM is low throughput while high throughput is major strength of SWATH-MS.

Reports describing the effect of Meth on expression and potential function(s) of galectins appear to be imprecise due to a variety of experimental models and experimental designs used. Here, we provide for the first time a systematic quantification of intracellular as well extracellular galectins.

Galectins are produced and secreted by many cell types and several important regulatory functions have been associated with their presence in circulation (Brinchmann et al. 2018; Johannes et al. 2018). Galectins belong to a group of cytoplasmic proteins that can be secreted to the surrounding milieu even without signal sequence for secretion. Popa et al. propose four mechanisms of secretion of galectins (Popa et al. 2018) out of which three are based on vesicle transport. Since we observe that in the presence of Meth, whether for shorter (CIM) or longer (MIM) time of exposure, secretion of all galectins is inhibited. Since we do not observe significant suppression of mRNA (see Fig. 3), transport of galectins to the extracellular space can be linked to deregulation of Rab proteins that are de-regulated upon Meth exposure (Macur et al. 2021) or other proteins involved in the regulation of vesicle-mediated transport.

Galectins are postulated to be involved in multiple regulatory processes via recognition of glycoconjugates, some of which are related to the regulation of the immune system by affecting T cells, macrophages, and B cells. As reported by Sato et al. (Sato et al. 2012), presentation of complex glycans on HIV-1 gp120 glycoprotein can be recognized by galectins-1, -3 , and -9 . Proposed mechanism postulates that these galectins might be involved in increasing HIV-1 infectivity and transmission via recognition of oligomannosyl polysaccharides, though their specificity and exact molecular mechanism is not entirely clear.

Our studies show that Meth has quite a profound effect on the expression of these three galectins in hMDM and this effect depends on the time of exposure, CIM vs. MIM. We suggest that these differences reflect the mechanism(s) of how macrophages try to cope with toxic insults such as presence of Meth. The molecular mechanism is not clear at this time and may include some epigenetically driven mechanisms to be explored in future studies.

Galectin-1. It was postulated that galectin-1 increases HIV-1 infectivity of T cells and macrophages through a mechanism involving an initial interaction with gp120 (Ouellet et al. 2015). Galectin-1 is the only galectin studied in the presence of illicit drugs (Reynolds et al. 2012a, b). In works of Reynolds group, morphine was used as a model, and the vast majority of results were acquired from experiments investigating gene expression. Our data presented in Fig. 4 show intracellular expression of galectin-1 in hMDM at the protein level showing significant suppression of this protein due to Meth exposure in already HIV-1-infected hMDM. Although prolonged exposure to Meth shows a slight rebounded expression, it still remains significantly lower than in the absence of Meth.

Galectin-3. It has been postulated that increase of galectin-3 is induced by expression of Tat protein mediated by the Sp-1 binding transcription factor. This conclusion was made based on an investigation involving several transformed cell lines (Fogel et al. 1999) but not primary human cells, such as T cells and macrophages, which are direct targets of HIV-1. Our data presented in Fig. 4 show a profound decreased expression of intracellular galectin-3 particularly after longer exposure to Meth as it was in MIM condition. This appears opposed to observations presented by Fogel et al. postulating that expression of galectin-3 is related to the expression of gene tat (Fogel et al. 1999). Otherwise, we would expect that transcription and protein level of Tat would be increased during active infection of 
Networks 1,1,1 Merged 3

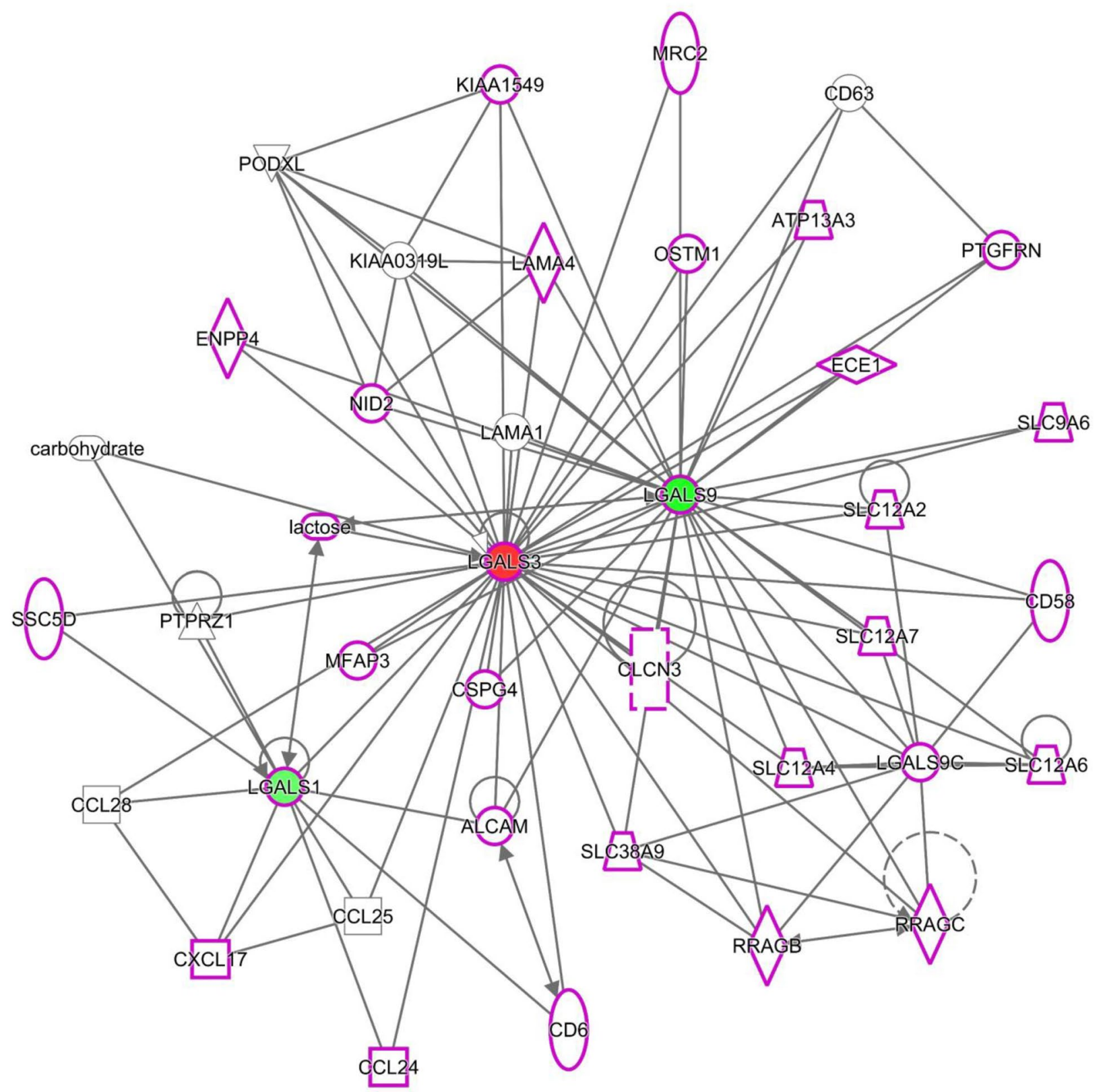

(c) 2000-2021 QIAGEN. All rights reserved.

Fig. 8 IPA network of galectins-1, -3 , and -9 interactions. Red represents upregulated genes while green represents downregulated genes. The intensity of colors is directly proportional to the fold change.

hMDM. Importantly, Lee and colleagues (Lee et al. 2002) did not observe an effect on SP-1 biding activity to DNA due to Meth injections into mice. Summarizing, it is not the first time when results from several studies show opposite results; therefore, galectins warrant further investigations because they are undoubtfully significant pathogenic factors in HIV-1 infections.
Purple shapes represent genes associated with lipid metabolism. The values are common number of genes between each observation

Galectin-9. The role of galectin-9 in viral infections, including HIV-1, appears multipurpose. Some reports suggest that galectin-9 plays a role as a negative regulator in T cells' immune response to viral infections (Merani et al. 2015), while others postulate that galectin- 9 is a potent mediator of HIV-1 transmission and reactivation (Abdel-Mohsen et al. 2016). It must be noted that all investigations reported to date are focused 
A

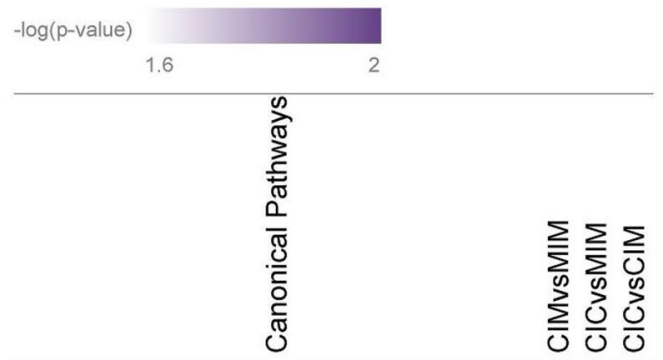

BEX2 Signaling Pathway

Th1 Pathway

Th1 and Th2 Activation Pathway

T Cell Exhaustion Signaling Pathway

Tumor Microenvironment Pathway

B

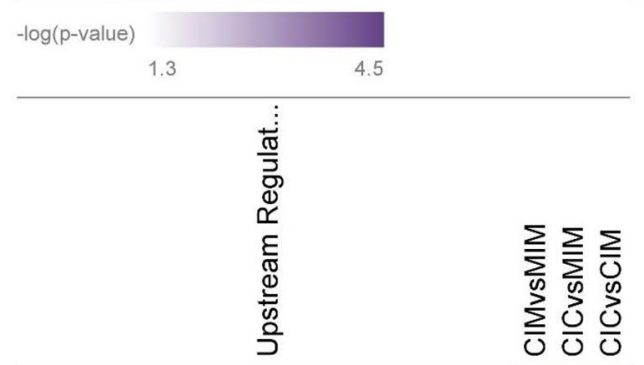

Pro-inflammatory Cytokine

lipopolysaccharide

HPS1

$\lg G$

COMP

miR-424-3p (miRNAs w/seed AAAACGU)

TH17 Cytokine

progesterone

goralatide

GCS-100

pilocarpine

SMARCA4

ERBB2

HIPK2

MDM4

decitabine

IFNG

eflornithine

GDNF

spironolactone

zymosan

ATG5

cadmium

salirasib

HDAC3

methamphetamine

tretinoin

Hif1

Ifn gamma

NRAS

CXCL8

aldosterone

TCF

TNF
4 Fig. 9 The results of the Ingenuity Pathways Core Analysis performed using $\log _{10}$ (Fold Change) values between CIC versus CIM, CIC versus MIM, and CIM versus MIM for galectins-1, -3 , and -9 quantified in the nanoLC-SWATH-MS experiment. (a) Canonical pathways in which the investigated galectins are involved. (b) Upstream regulators that might be activated or inhibited to explain expression changes of the studied galectins. The color intensity ranges from the lowest to highest $-\log$ ( $p$ value)

on $\mathrm{T}$ cells, while expression of galectin- 9 as a pathogenic and/ or regulatory factor was not investigated in macrophages. This makes our data presented in Fig. 5 a novel observation showing that when HIV-1-infected hMDMs are exposed to $100 \mu \mathrm{M}$ of Meth, expression of galectin-9 is significantly increased despite whether Meth is added prior to or after viral infection. We may speculate that increased expression of galectin-9 in hMDM will translate, in part, to increased extracellular levels of this lectin in cerebrospinal fluid (CSF) and/or peripheral blood. This hypothesis has yet to be tested, and the significance of galectin-9 expression intracellularly in macrophages is not clear. Another question is whether galectin-9 may contribute to higher infectivity of macrophages by HIV-1 while exposed to Meth.

\section{Conclusions}

Our data of comprehensive quantification of intra- and extracellular galectins uncover specific changes induced by Meth (Figs. 4 and 5). Interestingly, we did not observe significant effect of Meth at the RNAseq level. SWATH-MS analysis showed differerences in fold changes of galectin-1, -3 and -9 , between CIC, CIM and MIM conditions, but they were not statistically significant.This is most likely due to a lack of sensitivity required to quantify small changes. As expected, MRM measurements showed more significant differences. Some differences are very small and exhibit significance close to borderline. Immune-cytostaining showed quite even distribution of galectins throughout cytoplasm with some overlap with lysosomes stained with LysoTracker. Further bioinformatic investigations show pro-inflammatory phenotype of hMDM and suggest a possibility of a much broader effect particularly in the local milieu of macrophages residing in tissues such as the brain (Figs. 8 and 9).

Thus, our study provides the first comprehensive quantification of three galectins representing three types of these proteins and should be considered as a foundation for future studies such as latency of HIV-1 infection. Further bioinformatic investigation of metabolic pathways combined with various experimental designs might be critical in deciphering the exact role of these important regulators of the innate immune system as potential biomarkers of disease progression and efficacy of therapies. 
Supplementary information The online version contains supplementary material available at https://doi.org/10.1007/s13365-021-01025-4.

Acknowledgements The authors would like to thank Ms. Robin Taylor for her assistance in editing of text and preparation of graphics for publication.

The mass spectrometry instrumentation available in the Mass Spectrometry and Proteomics Core Facility of the UNMC was used to perform MS analyses. The UNMC Mass Spectrometry and Proteomics Core Facility, which is administrated through the Office of the Vice Chancellor for Research, and is supported by state funds from the Nebraska Research Initiative (NRI).

The RNAseq was performed at the UNMC Genomics Core Facility, which receives partial support from the Nebraska Research Network in Functional Genomics NE-INBRE P20GM103427-14 and the Fred \& Pamela Buffett Cancer Center (P30CA036727), and the resulting data were analyzed using bioinformatic pipeline from the UNMC Bioinformatics and Systems Biology Core.

Authors' contribution PC was principal investigator of this study. PC and $\mathrm{HSF}$ conceived and designed the experiments. $\mathrm{KG}, \mathrm{KM}, \mathrm{SZ}, \mathrm{NH}$, and AS performed the experimental part. KM and LZ performed data analysis. Interpretation of results was performed by $\mathrm{PC}, \mathrm{KG}, \mathrm{KM}$, and HSF. PC, KG, and KM wrote the paper. All co-authors reviewed and approved final version of manuscript.

Funding Financial support was provided by National Institutes of Health grants: R01 DA043258 and P30 MH062261.

Data availability The mass spectrometry datasets used in this study were deposited to PRIDE (DDA and SWATH data - accession number PXD023291) or the PeptideAtlas PASSEL (MRM data - accession number PASS01591) repositories, which are both members of the ProteomeXChange Consortium. The RNAseq datasets have deposited in NCBI GEO and are available with accession number GSE160323.

\section{Declarations}

Competing interests The authors declare no competing interests.

Open Access This article is licensed under a Creative Commons Attribution 4.0 International License, which permits use, sharing, adaptation, distribution and reproduction in any medium or format, as long as you give appropriate credit to the original author(s) and the source, provide a link to the Creative Commons licence, and indicate if changes were made. The images or other third party material in this article are included in the article's Creative Commons licence, unless indicated otherwise in a credit line to the material. If material is not included in the article's Creative Commons licence and your intended use is not permitted by statutory regulation or exceeds the permitted use, you will need to obtain permission directly from the copyright holder. To view a copy of this licence, visit http://creativecommons.org/licenses/by/4.0/.

\section{References}

Abdel-Mohsen M, Chavez L, Tandon R, Chew GM, Deng X, Danesh A, Keating S, Lanteri M, Samuels ML, Hoh R, Sacha JB, Norris PJ, Niki T, Shikuma CM, Hirashima M, Deeks SG, Ndhlovu LC, Pillai SK (2016) Human Galectin-9 Is a Potent Mediator of HIV Transcription and Reactivation. PLoS Pathog 12:e1005677.
Barbosa DJ, Capela JP, Feio-Azevedo R, Teixeira-Gomes A, Bastos Mde L, Carvalho F (2015) Mitochondria: key players in the neurotoxic effects of amphetamines. Arch Toxicol 89:1695-1725

Bissa B, Beedle AM, Govindarajan R (2016) Lysosomal solute carrier transporters gain momentum in research. Clin Pharmacol Ther 100:431-436

Bortell N, Morsey B, Basova L, Fox HS, Marcondes MC (2015) Phenotypic changes in the brain of SIV-infected macaques exposed to methamphetamine parallel macrophage activation patterns induced by the common gamma-chain cytokine system. Front Microbiol 6:900

Brinchmann MF, Patel DM, Iversen MH (2018) The Role of Galectins as Modulators of Metabolism and Inflammation. Mediators Inflamm 2018:9186940

Burns A, Ciborowski P (2016) Acute exposure to methamphetamine alters TLR9-mediated cytokine expression in human macrophage. Immunobiology 221:199-207

Farrah T, Deutsch EW, Kreisberg R, Sun Z, Campbell DS, Mendoza L, Kusebauch U, Brusniak MY, Huttenhain R, Schiess R, Selevsek N, Aebersold R, Moritz RL (2012) PASSEL: the PeptideAtlas SRMexperiment library. Proteomics 12:1170-1175

Fogel S, Guittaut M, Legrand A, Monsigny M, Hebert E (1999) The tat protein of HIV-1 induces galectin-3 expression. Glycobiology 9:383-387

Haverland NA, Fox HS, Ciborowski P (2014) Quantitative proteomics by SWATH-MS reveals altered expression of nucleic acid binding and regulatory proteins in HIV-1-infected macrophages. J Proteome Res 13:2109-2119

Ikeda K, Quertermous T (2004) Molecular isolation and characterization of a soluble isoform of activated leukocyte cell adhesion molecule that modulates endothelial cell function. J Biol Chem 279:55315-55323

Johannes L, Jacob R, Leffler H (2018) Galectins at a glance. J Cell Sci 131

Kraft-Terry SD, Buch SJ, Fox HS, Gendelman HE (2009) A coat of many colors: neuroimmune crosstalk in human immunodeficiency virus infection. Neuron 64:133-145

Laederach A, Reilly PJ (2005) Modeling protein recognition of carbohydrates. Proteins 60:591-597

Lee WY, Wang CJ, Lin TY, Hsiao CL, Luo CW (2013) CXCL17, an orphan chemokine, acts as a novel angiogenic and anti-inflammatory factor. Am J Physiol Endocrinol Metab 304:E32-40

Lee YW, Son KW, Flora G, Hennig B, Nath A, Toborek M (2002) Methamphetamine activates DNA binding of specific redox-responsive transcription factors in mouse brain. J Neurosci Res 70:82-89

Liang H, Wang X, Chen H, Song L, Ye L, Wang SH, Wang YJ, Zhou L, Ho WZ (2008) Methamphetamine enhances HIV infection of macrophages. Am J Pathol 172:1617-1624

Lyons A, Pitts M, Grierson J (2013) Methamphetamine use in a nationwide online sample of older Australian HIV-positive and HIVnegative gay men. Drug Alcohol Rev 32:603-610

MacLean B, Tomazela DM, Shulman N, Chambers M, Finney GL, Frewen B, Kern R, Tabb DL, Liebler DC, MacCoss MJ (2010) Skyline: an open source document editor for creating and analyzing targeted proteomics experiments. Bioinformatics 26:966-968

Macur K, Zieschang S, Lei S, Morsey B, Jaquet S, Belshan M, Fox HS, Ciborowski P (2021) SWATH-MS and MRM: Quantification of Ras-related proteins in HIV-1 infected and methamphetamineexposed human monocyte-derived macrophages (hMDM). Proteomics: e2100005.

Merani S, Chen W, Elahi S (2015) The bitter side of sweet: the role of Galectin-9 in immunopathogenesis of viral infections. Rev Med Virol 25:175-186

Mitchell SJ, Morris SR, Kent CK, Stansell J, Klausner JD (2006) Methamphetamine use and sexual activity among HIV-infected 
patients in care-San Francisco, 2004. AIDS Patient Care STDS 20:502-510

Naderi A, Liu J, Bennett IC (2010) BEX2 regulates mitochondrial apoptosis and $\mathrm{G} 1$ cell cycle in breast cancer. Int $\mathrm{J}$ Cancer 126:1596-1610

Niu M, Morsey B, Lamberty BG, Emanuel K, Yu F, Leon-Rivera R, Berman JW, Gaskill PJ, Matt SM, Ciborowski PS, Fox HS (2020) Methamphetamine Increases the Proportion of SIV-Infected Microglia/Macrophages, Alters Metabolic Pathways, and Elevates Cell Death Pathways: A Single-Cell Analysis. Viruses 12

Ouellet M, St-Pierre C, Tremblay MJ, Sato S (2015) Effect of galectins on viral transmission. Methods Mol Biol 1207:397-420

Paclik D, Werner L, Guckelberger O, Wiedenmann B, Sturm A (2011) Galectins distinctively regulate central monocyte and macrophage function. Cell Immunol 271:97-103

Pantalone DW, Bimbi DS, Holder CA, Golub SA, Parsons JT (2010) Consistency and change in club drug use by sexual minority men in New York City, 2002 to 2007. Am J Public Health 100:1892-1895

Papageorgiou M, Raza A, Fraser S, Nurgali K, Apostolopoulos V (2019) Methamphetamine and its immune-modulating effects. Maturitas 121:13-21

Parikh NU, Aalinkeel R, Reynolds JL, Nair BB, Sykes DE, Mammen MJ, Schwartz SA, Mahajan SD (2015) Galectin-1 suppresses methamphetamine induced neuroinflammation in human brain microvascular endothelial cells: Neuroprotective role in maintaining blood brain barrier integrity. Brain Res 1624:175-187

Passaro RC, Pandhare J, Qian HZ, Dash C (2015) The Complex Interaction Between Methamphetamine Abuse and HIV-1 Pathogenesis. J Neuroimmune Pharmacol 10:477-486

Perez-Riverol Y, Csordas A, Bai J, Bernal-Llinares M, Hewapathirana S, Kundu DJ, Inuganti A, Griss J, Mayer G, Eisenacher M, Perez E, Uszkoreit J, Pfeuffer J, Sachsenberg T, Yilmaz S, Tiwary S, Cox J, Audain E, Walzer M, Jarnuczak AF, Ternent T, Brazma A, Vizcaino JA (2019) The PRIDE database and related tools and resources in 2019: improving support for quantification data. Nucleic Acids Res 47:D442-D450

Popa SJ, Stewart SE, Moreau K (2018) Unconventional secretion of annexins and galectins. Semin Cell Dev Biol 83:42-50
Reynolds JL, Law WC, Mahajan SD, Aalinkeel R, Nair B, Sykes DE, Mammen MJ, Yong KT, Hui R, Prasad PN, Schwartz SA (2012a) Morphine and galectin-1 modulate HIV-1 infection of human monocyte-derived macrophages. J Immunol 188:3757-3765

Reynolds JL, Law WC, Mahajan SD, Aalinkeel R, Nair B, Sykes DE, Yong KT, Hui R, Prasad PN, Schwartz SA (2012b) Nanoparticle based galectin-1 gene silencing, implications in methamphetamine regulation of HIV-1 infection in monocyte derived macrophages. J Neuroimmune Pharmacol 7:673-685

Reynolds JL, Mahajan SD, Sykes DE, Schwartz SA, Nair MP (2007) Proteomic analyses of methamphetamine (METH)-induced differential protein expression by immature dendritic cells (IDC). Biochim Biophys Acta 1774:433-442

Saito M, Terada M, Kawata T, Ito H, Shigematsu N, Kromkhun P, Yokosuka M, Saito TR (2008) Effects of single or repeated administrations of methamphetamine on immune response in mice. Exp Anim 57:35-43

Sato S, Ouellet M, St-Pierre C, Tremblay MJ (2012) Glycans, galectins, and HIV-1 infection. Ann N Y Acad Sci 1253:133-148

Schwarz FP, Puri KD, Bhat RG, Surolia A (1993) Thermodynamics of monosaccharide binding to concanavalin A, pea (Pisum sativum) lectin, and lentil (Lens culinaris) lectin. J Biol Chem 268:7668-7677

Shahbaz S, Dunsmore G, Koleva P, Xu L, Houston S, Elahi S (2020) Galectin-9 and VISTA Expression Define Terminally Exhausted T Cells in HIV-1 Infection. J Immunol 204:2474-2491

Talloczy Z, Martinez J, Joset D, Ray Y, Gacser A, Toussi S, Mizushima N, Nosanchuk JD, Goldstein H, Loike J, Sulzer D, Santambrogio L (2008) Methamphetamine inhibits antigen processing, presentation, and phagocytosis. PLoS Pathog 4:e28

Vladoiu MC, Labrie M, St-Pierre Y (2014) Intracellular galectins in cancer cells: potential new targets for therapy (Review). Int $\mathbf{J}$ Oncol 44:1001-1014

Wisniewski JR, Zougman A, Nagaraj N, Mann M (2009) Universal sample preparation method for proteome analysis. Nat Methods $6: 359-362$

Publisher's Note Springer Nature remains neutral with regard to jurisdictional claims in published maps and institutional affiliations. 